\title{
BMJ Open A mixed methods survey of social anxiety, anxiety, depression and wig use in alopecia
}

\author{
Kerry Montgomery, ${ }^{1}$ Caroline White, ${ }^{2}$ Andrew Thompson ${ }^{1}$
}

To cite: Montgomery K, White C Thompson A. A mixed methods survey of social anxiety, anxiety, depression and wig use in alopecia. BMJ Open 2017;7:e015468. doi:10.1136/ bmjopen-2016-015468

- Prepublication history and additional material are available. To view please visit the journal (http://dx.doi.org/10.1136/ bmjopen-2016-015468).

Received 20 December 2016 Revised 21 March 2017 Accepted 22 March 2017

\section{(a) CrossMark}

${ }^{1}$ Department of Psychology, University of Sheffield, Sheffield, UK

${ }^{2}$ Dermatopharmacology Unit, University of Manchester,

Manchester, Greater

Manchester, UK

Correspondence to

Kerry Montgomery;

kmontgomery1@sheffield.ac.uk

\section{ABSTRACT}

Objectives This study aimed to examine levels of social anxiety, anxiety and depression reported by people with alopecia as a result of a dermatological condition and associations with wig use. The study also sought to report on experiences of wearing wigs in social situations and the relationship with social confidence.

Design A cross-sectional survey was sent by email to the Alopecia UK charity mailing list and advertised on social media.

Participants Inclusion criteria were a diagnosis of alopecia, aged 13 or above and sufficient English to complete the survey. Exclusion criteria included experiencing hair loss as a result of chemotherapy treatment or psychological disorder. Participants $(n=338)$ were predominantly female $(97.3 \%)$, Caucasian $(93.5 \%)$ and aged between 35 and 54 years (49.4\%) with a diagnosis of alopecia areata $(82.6 \%)$.

Main outcome measures The Social Phobia Inventory measured symptoms of social anxiety, and the Hospital Anxiety and Depression Scale was used to measure symptoms of anxiety and depression. Survey questions were designed to measure the use of wigs. Open-ended questions enabled participants to comment on their experiences of wearing wigs.

Results Clinically significant levels of social anxiety $(47.5 \%)$, anxiety (35.5\%) and depression (29\%) were reported. Participants who reported worries about not wearing a wig reported significantly higher levels of depression: $t(103)=3.40, p \leq 0.001$; anxiety: $t(109)=4.80$, $\mathrm{p} \leq 0.001$; and social anxiety: $\mathrm{t}(294)=3.89, \mathrm{p} \leq 0.001$. Wearing wigs was reported as increasing social confidence; however, the concealment it afforded was also reported as both reducing fear of negative evaluation and maintaining anxiety.

Discussion Overall, $46 \%$ of participants reported that wearing a wig had a positive impact on their everyday life with negative experiences related to fears of the wig being noticed. Psychological interventions alongside wig provision would be beneficial for people living with alopecia.

\section{INTRODUCTION}

For many people hair is a central aspect of appearance and self-image; therefore, hair loss can have a negative impact on self-esteem, body image and confidence. ${ }^{12}$ While hair loss may have few physical health
Strengths and limitations of this study

- This is the first study to examine National Health Service wig provision and how people living with alopecia finance wigs.

- Validated measures of social anxiety, anxiety and depression were used to determine levels of distress.

- Survey questions investigated the experience of wearing wigs.

- The sample was predominantly female and Caucasian; therefore, further research is needed to examine experiences of men and cultural differences in alopecia.

- Participants recruited from Alopecia UK may have been experiencing higher levels of distress than the general population, having accessed support by the charity.

consequences, living with a condition that affects appearance can have a significant impact on everyday functioning. ${ }^{3-6}$ Studies have shown that people living with alopecia are at a higher risk of developing depression, anxiety and social phobia than the general population. $^{78}$

Higher levels of body dissatisfaction and preoccupation with appearance have been reported in men and women living with alopecia, relative to controls, ${ }^{79} 10$ with $40 \%$ of women reporting marital problems as a consequence. ${ }^{3}$ Perception of hair loss has a significant impact on quality of life, accounting for $35 \%$ of the variance. ${ }^{11}$

Wearing a wig to conceal hair loss is an important coping strategy for many individuals $^{12-15}$ giving people the confidence to return to work and engage in social activity. ${ }^{14}$ We have known since the time of Goffman's seminal work on stigmatisation ${ }^{16}$ that people may choose to conceal attributes they believe will be discredited by others. Recent studies indicate that alopecia areata can be associated with higher levels of perceived stigmatisation than concealable conditions such as mental health conditions. ${ }^{17}$ Wearing a wig might reduce an individuals' perception of a 
discrepancy between cultural norms regarding the appearance of the hair and their own appearance. However, wearing a wig might not be an ideal solution as the fear of being 'discredited' should the wig be noticed remains. ${ }^{16}$

Despite wigs being an important coping strategy for people living with alopecia, many people experience difficulties accessing wig prescriptions via the National Health Service (NHS). Some NHS trusts report that alopecia is a 'cosmetic issue'; therefore, the financial burden of wigs falls with the patient. Further cuts in dermatology could mean more patients have difficulties accessing wigs, which may have significant psychosocial consequences for people living with alopecia. Access to psychological support within dermatology is limited, ${ }^{18}$ and currently there are no psychological interventions available to target the specific needs of this population. ${ }^{19}$ The need for psychological interventions for alopecia has been emphasised by patients and clinicians. ${ }^{20}$

The current mixed methods study aims to examine (1) levels of social anxiety, anxiety and depression reported by people living with alopecia, (2) associations between wig behaviours and psychosocial distress and (3) experiences of wearing wigs in social situations. The current study will also investigate how participants access wigs and the financial implications of wearing wigs.

\section{Method}

A cross-sectional survey incorporating validated measures of social anxiety, depression and anxiety, questions on wig usage, financing wigs, and open-ended questions regarding experiences of wearing wigs in social situations was sent by email to the Alopecia UK mailing list and advertised on social media. Ethical approval for the study was gained from the University of Sheffield Psychology Department Ethics Committee. Inclusion criteria were: a diagnosis of alopecia as a result of a dermatological condition, aged 13 or above and sufficient English proficiency to complete the survey. Participants aged under 16 years of age were advised to complete the questionnaire with a parent or guardian. Exclusion criteria included experiencing hair loss as a result of chemotherapy treatment or primary psychological disorder (eg, trichotillomania).

\section{Measures}

\section{Social anxiety}

The Social Phobia Inventory (SPIN) ${ }^{21}$ is a 17 -item self-report questionnaire to measure various elements of social anxiety (fear in social situations, avoidance of social situations/performances and physiological symptoms in social situations). Connor et $a l^{21}$ reported a mean score of 41 on the SPIN for individuals with social phobia, compared with a score of 12 in a non-psychiatric population. A score of 19 or above indicates symptoms of social anxiety with $79 \%$ diagnostic accuracy. ${ }^{21}$

\section{Depression}

The nine-item self-report Patient Health Questionnaire ${ }^{22}$ was used to measure symptoms of depression experienced over the last 2 weeks. A mean score of 3 has been reported by healthy controls, in comparison with a score of 17 in people diagnosed with depression. ${ }^{22}$ A cut-off score of $\geq 10$ was used to identify individuals experiencing depression ( $88 \%$ specificity and $88 \%$ sensitivity). ${ }^{23}$

\section{Anxiety}

The Generalised Anxiety Disorder Questionnaire ${ }^{24}$ is a seven-item self-report scale to measure generalised anxiety symptoms over the last 2 weeks. A mean score of 4.9 has been reported in healthy controls, in comparison with a score of 14 in people living with generalised anxiety. ${ }^{24}$ A cut-off score of $\geq 8$ was used to identify individuals experiencing anxiety. ${ }^{25}$

\section{Survey questions}

A number of survey questions were included in which participants were asked to report on the situations in which they would choose to wear a wig (eg, social situations, dating, work/university/school) and if they had any worries about financing or not wearing wigs. Open-ended questions were asked to elicit experiences of wearing wigs in social situations. Questions included: Has wearing a wig had any impact on your confidence during social situations?, Do you have any worries about not wearing a wig? and How has wearing a wig affected your everyday life?

\section{Analysis}

Statistical analysis was carried out using SPSS V.22. ${ }^{26}$ Descriptive statistics were used to provide information on (1) demographic and clinical variables, (2) percentage of people wearing wigs and the situations in which people would choose to wear wigs, (3) wig funding and (4) clinically significant levels of social anxiety, depression and anxiety. Associations were tested between demographic variables, wig behaviour (eg, situations in which people would choose to wear wigs) and social anxiety, depression and anxiety using independent samples t-tests and Pearson correlation as appropriate. An alpha level of 0.05 was used for all statistical tests.

Qualitative responses were analysed using qualitative content analysis. ${ }^{27}$ Qualitative Content Analysis is a systematic text analysis technique that preserves the advantages of quantitative analysis, allowing frequencies of data to be reported while also providing a method of interpreting participant experiences of wearing wigs during social situations. $^{27}$

An inductive approach to data analysis was used to determine emergent themes generated through examination of participant's responses to specific questions. The data were analysed based on the wording used by participants to describe their experiences and organised into higher order themes. For example, the response that wigs 'improved confidence' and participants 'felt more able to go out' was coded into the themes 'improved confidence' and 'positive experience of wearing a wig'. Frequency of the occurrence of themes across participants was recorded by the first and second authors. Inter-rater reliability 


\begin{tabular}{|c|c|c|}
\hline & $\mathbf{n}$ & $\%$ \\
\hline \multicolumn{3}{|l|}{ Type of alopecia } \\
\hline Alopecia areata & 114 & 33.7 \\
\hline Universalis & 106 & 31.4 \\
\hline Totalis & 59 & 17.5 \\
\hline Frontal fibrosing & 7 & 2.1 \\
\hline Androgenic & 8 & 2.4 \\
\hline Male pattern baldness & 3 & 0.9 \\
\hline Scarring & 3 & 0.9 \\
\hline Female pattern baldness & 2 & 0.6 \\
\hline Lichen planopilaris & 1 & 0.3 \\
\hline Folliculitis decalvans & 1 & 0.3 \\
\hline Chemically induced & 1 & 0.3 \\
\hline Unknown & 32 & 9.5 \\
\hline \multicolumn{3}{|l|}{ Gender } \\
\hline Female & 329 & 97.3 \\
\hline Male & 5 & 1.5 \\
\hline No response & 1 & 0.3 \\
\hline \multicolumn{3}{|l|}{ Ethnicity } \\
\hline White (Caucasian) & 316 & 93.5 \\
\hline Asian (British Asian) & 10 & 3.0 \\
\hline Black (Black British) & 5 & 1.5 \\
\hline Other & 4 & 1.2 \\
\hline No response & 1 & 0.3 \\
\hline
\end{tabular}

(98\%) was established by determining any differences in coding between the first and second authors.

\section{RESULTS}

\section{Participants}

Participants $(\mathrm{n}=338)$ were predominantly female $(97.3 \%)$ and Caucasian $(93.5 \%)$. All participants lived in the UK. The majority of the sample were aged between 35 and 54 years $(49.4 \%)(13-17$ years $1.5 \%, 18-25$ years $8 \%$, 26-34 years $13.6 \%$, 55-64 years $18.6 \%$ and 65 years and over $8.9 \%$ ). Eleven different types of alopecia were reported across the sample (table 1 ), and the majority of the sample comprised of alopecia areata $(82.6 \%)$. Of the respondents, $10.7 \%$ were receiving medical treatment for hair loss including steroid injections, spironolactone and topical treatments.

The majority of participants chose to wear wigs most or all of the time $(76 \%$ ) (table 2 ). The most common type of wigs worn by participants were acrylic (acrylic monofilament $39.3 \%$, acrylic lace front $27.5 \%$ and acrylic wefted wig $14.8 \%$ ). The majority of participants did not obtain NHS wig prescriptions $(50.6 \%)$ out of which $11.2 \%$ of participants had been told that they were not eligible and $22.5 \%$ had never enquired about it. Of the participants who claimed NHS wig prescriptions (46.1\%), $11.5 \%$

Table 2 Wig use of participants $(n=338)$

$\%$ of people wearing

\begin{tabular}{ll} 
How often do you wear a wig? & a wig \\
\hline Never & 23.6 \\
Occasionally & 16.6 \\
Most of the time & 26 \\
\hline All the time (excluding the night) & 44.1 \\
\hline All the time (including the night) & 5.9 \\
\hline
\end{tabular}

claimed one per year, 26\% claimed two per year and $8.6 \%$ claimed more than two each year.

\section{Social anxiety, anxiety and depression}

Clinically significant levels of anxiety (score $\geq 8$ ) were reported by $35.5 \%$ of participants, with $40.5 \%$ reporting no symptoms, $14.5 \%$ reporting mild symptoms (score $7-9), 13.5 \%$ reporting moderate symptoms and $15 \%$ reporting severe symptoms of anxiety. Clinically significant levels of depression (score $\geq 10$ ) were reported by $29 \%$ of the sample, with $43.6 \%$ reporting no symptoms, $27.6 \%$ reporting mild symptoms (score 5-9), 14.3\% reporting moderate symptoms (score 10-14), 8.1\% reporting moderately severe symptoms and $6.6 \%$ reporting severe symptoms of depression. Clinically significant symptoms of social anxiety (score $\geq 19$ ) were reported by $47.5 \%$ of the sample, with $23.4 \%$ reporting mild symptoms, $11.1 \%$ reporting moderate symptoms, $7.2 \%$ reporting severe symptoms and $6.6 \%$ reporting very severe symptoms. Participants reporting worries about not wearing a wig reported significantly higher levels of depression: $\mathrm{t}(103)=3.40, \mathrm{p} \leq 0.001 ;$ anxiety: $\mathrm{t}(109)=4.80, \mathrm{p} \leq 0.001$; and social anxiety: $\mathrm{t}(294)=3.89, \mathrm{p} \leq 0.001$.

\section{Wig use}

Participants were asked when they wear a wig across a number of everyday activities (table 3). The majority of participants reported wearing a wig to socialise $(86.7 \%)$ and $66.3 \%$ of respondents reported they would not feel confident leaving the house without a wig. Of the participants who purchased wigs via NHS prescription, $28.1 \%$ reported that they would be unable to afford their wig privately and $65.1 \%$ of the sample reported worry about affording new wigs.

\begin{tabular}{ll}
\hline $\begin{array}{l}\text { Table } 3 \text { Situations in which people would wear a wig } \\
(\mathrm{n}=338)\end{array}$ \\
\hline $\begin{array}{l}\text { Situations when you would } \\
\text { wear a wig }\end{array}$ & $\%$ of people wearing a wig \\
\hline Meeting new people & 78.4 \\
Dating & 61.2 \\
Socialising & 86.7 \\
Work/school/university & 76.3 \\
All the time & 55.9 \\
\hline
\end{tabular}




\section{Qualitative findings}

A summary of responses to open-ended survey responses is reported below.

Has wearing a wig had any impact on confidence during social situations?

Three hundred and thirteen participants responded to the question on the impact of wigs on confidence in social situations. Of responses, $26 \%$ reported a positive impact of wearing a wig. The positive impact was related to the reduced likelihood of comments regarding hair loss in 23\% of participants and feeling more confident going out in public (32\%).

Interacting with people who I've never met before, without a wig they may wonder why I have no hair, it is not, the norm to have patchy bald head. Hate the idea people will talk about me, or feel I have a more serious illness.

At different times in my life a wig has been a survival tool for me.

Participants also reported that wearing a wig could have a negative impact on confidence during social situations (43\%). These responses were categorised into two main themes: worries about others knowing it was a wig $(47 \%)$ and concerns about the wig coming off or discomfort (39\%). Wearing a wig also led to reduced activity $(41 \%)$ in particular sports were avoided due to concerns about having to take off the wig.

Even 10 years on, I still wonder if people can tell I wear a wig and especially when I meet new people. It has stopped me going to places or trying new activities.

Difficulty adjusting to a change in appearance when wearing a wig was reported with participants describing feeling disconnected from their appearance in a wig.

No matter how attractive I look in a wig I feel like a sham. I feel the real bald me is not attractive at all.

For some participants $(7.8 \%)$ wearing a wig had no impact on their confidence, which was due to positive experiences of wearing a wig. Other responses were not coded as participants had simply responded 'no', which was interpreted as 'no impact', but it was unclear if this was related to a positive or negative impact of wigs.

\section{How has wearing a wig affected your everyday life?}

Of the 299 responses, 23\% of participants reported that wearing a wig improved their confidence/self-esteem in everyday life.

It's improved my confidence as I avoided harsh overhead lights in retail stores as it emphasised my thinning hair particularly distressing when I saw my reflection in a mirror. Now I can walk past a mirror with harsh lighting overhead and check my hair and smile!
It's given me a lot more confidence in just leaving the house and being in front of other people.

Negative views of appearance without a wig were reported $(14 \%)$.

Don't like anyone seeing me without it, feel naked. I feel a lot less feminine without hair.

Participants reported negative reactions from others if they were not wearing a wig (9.6\%), suggesting wigs were used as a coping strategy to manage negative reactions.

It is an uncomfortable but manageable part of my life. It means that I don't get as many pitying glances from strangers.

I feel more confident to go out with my children, it becomes incredibly tiring been stared at and shouted at if I ever left the house without my wig on.

Overall, $46.6 \%$ of participants reported that wearing a wig had a positive impact on their everyday life, reducing the likelihood of negative reactions while improving confidence with regards to appearance.

Participants also reported problems with wigs that had a negative impact on everyday life (49\%; subthemes included: wigs coming off, expense, quality and fitting).

Wearing a wig is a way of life to me as I've never had hair, but always dreamed of being normal. Not worrying if it's going to fall off or move.

Every time I step outside my front door I think about weather conditions, who I might meet, if anyone is going to stand too close to me, what they will see, what they will think.

In addition, wearing wigs reduced social activity $(33 \%)$ in particular; dating, meeting new people and exercise were situations in which participants experienced problems.

It's depressing every morning having to wake up and put my wig on. When I sleep with someone new in my room I don't take my wig off.

Constant worry that someone/child will randomly pull the hair, or know it is a wig and make comments. Still anxious when out, but at least I now leave the house.

Going swimming is difficult I don't like swimming hats, and use an old wig, but can't dive in a pool or swim under water with a wig on, constantly scared of losing wig in water, but love to swim.

Participants reported feeling more self-conscious about having to wear a wig $(17 \%)$. Self-consciousness appeared to arise as a result of concerns about others finding out they were wearing a wig $(22 \%)$.

The NHS wigs look like wigs so I always imagine people are saying 'Look at her wearing that stupid wig'. I only go out if absolutely necessary and avoid public places as often as I can. I FEEL LIKE A FREAK. I've had bad experiences at work and socially where someone took my wig for a bet/ cos they were drunk. 


\section{Worries about not wearing a wig}

Participants reported worries about not wearing a wig $(40.5 \%)$ due to the reactions of others to hair loss (staring $42 \%$ and comments $18 \%$ ).

People as a rule have hair, why would I want people to stare and comment?

Specific concerns about not wearing a wig included worries others would assume they had cancer $(n=13)$.

People assume I have cancer. I look strange.

Self-conscious emotions and negative self-appraisals of appearance also led to worries about not wearing a wig $(19 \%)$.

I am worried people will see how ugly I really am if they see me bald.

\section{DISCUSSION}

The first aim of the current study was to investigate the prevalence of social anxiety, anxiety and depression in people living with alopecia. Social anxiety was commonly reported by the participants in this study with $47.5 \%$ indicating clinically significant levels of social anxiety. While few quantitative studies have examined the prevalence of social anxiety in people living with alopecia specifically, qualitative studies suggest that alopecia can have a significant impact on social functioning. ${ }^{2-41314}$ The prevalence of anxiety and depression reported are consistent with previous studies ${ }^{2}$ and highlights the importance of identifying coping strategies to help people living with alopecia to manage psychological distress.

The current study also aimed to examine the relationship between psychological variables and wig usage. Worry about not wearing a wig was related to higher levels of depression, anxiety and social anxiety, and with $65 \%$ of participants reporting worry about affording new wigs, it suggests the financial burden of affording wigs could lead to additional distress in patients already reporting significant levels of social anxiety, depression and anxiety. This finding has important implications in the current economic climate and suggests changes in NHS wig provision could have negative psychosocial consequences should people with alopecia no longer be able to access wig prescriptions.

For some people living with alopecia, this study clearly shows that wearing a wig is an important coping strategy, helping people to manage negative reactions from others and improving confidence to engage in social activity. However, despite wigs being viewed as an important coping strategy, only $46.7 \%$ of the participants who chose to wear wigs accessed NHS wig prescriptions, with some NHS trusts failing to provide any wig provision for patients with alopecia.

The final aim of the current study was to explore participants' experiences of wearing wigs in social situations. The current findings suggest that the relationship between wigs and social confidence is complex, and wigs alone do not appear to reduce social distress for a large number of patients. The current findings indicate that people living with alopecia face a difficult choice in social situations, negative reactions as a result of hair loss being visible or negative reactions as a result of wearing a wig. Managing the noticeability of wigs appeared to lead to significant negative interpersonal consequences, including avoidance of social situations and exercise. Despite generating anxiety and causing discomfort, participants were still choosing to wear wigs suggesting wigs were seen as the 'lesser of two evils' given worries about reactions of others to hair loss. These findings are consistent with previous studies and suggest that wearing a wig does not signify an end point to managing social distress for people living with alopecia. ${ }^{13} 14$

Critical self-appraisals of appearance without a wig ('freak, alien, ugly') appeared to be a contributing factor to wearing a wig, as wigs improved confidence in social situations through reducing appearance concern. Hair loss was viewed as a negative attribute with participants reporting experiences of stigma including comments and staring, and perception of hair loss as a sign of illness. Hair loss is reported as confirmation of identity for cancer patients, ${ }^{28}$ which has social implications for people living with alopecia, in that they are often misidentified as 'ill'.

Negative reactions from others represent threats to an individual's desire to be valued and accepted by others. ${ }^{16}$ Reactions to social rejection may be prosocial, involving an increased desire to be socially accepted; antisocial, whereby the individual attempts to defend themselves or express anger following rejection; and avoidant, ${ }^{29}$ in which an individual may seek to avoid further rejection that could involve reduced social engagement. Wearing a wig may therefore be seen as a strategy to increase social acceptance and to avoid rejection. Indeed, Goffman's seminal work on stigma ${ }^{16}$ suggests that individuals may choose to conceal attributes they believe will be discredited by others, in this case hair loss; however, the consequence of this is continued concealment and anxiety about being 'found out'.

There are several limitations of the current study that should be noted. First, the sample is predominantly female and Caucasian; therefore, further research is needed to explore the experiences of men and cultural differences in experiences of alopecia. Second, a number of responses could not be coded into themes as the responses did not relate to the question suggesting some participants may have experienced problems interpreting the questions. Third, participants were recruited from Alopecia UK; therefore, some participants may have been experiencing higher levels of distress than the general population having accessed support via the charity. Finally, given that the participants were recruited from the Alopecia UK mailing list and social media, it is not possible to ascertain a response rate in the current study.

The current findings have important implications when providing support to people living with alopecia and suggest that wigs appear to be much more than 
a 'cosmetic' tool and are used to reduce potential experiences of stigmatisation and improve social confidence. Given the significant negative social consequences that could arise should wigs no longer be an option, the current findings should be considered when funding decisions are made regarding wig provision for patients with alopecia. Negative experiences of wearing wigs are related to fears of the wig being noticed and hair loss being discovered; therefore, psychological interventions, particularly those targeting self-conscious emotions such as shame and social anxiety, would be beneficial for people living with alopecia alongside wig provision. To date, there are no studies examining the effectiveness of psychological interventions specific to people living with alopecia; however, approaches such as cognitivebehavioural therapy and compassion focused therapy may be beneficial in targeting processes underlying social anxiety. Attentional training techniques such as mindfulness may also be beneficial to reduce preoccupation with appearance-related thoughts. ${ }^{30}$

Supplementary data

The authors would like to thank Alopecia UK for their support in recruitment for this study.

\section{REFERENCES}

1. Thompson AR. Skin conditions. Encyclopaedia of body image and human appearance:738-44.

2. Tucker P. Bald is beautiful? The psychosocial impact of alopecia areata. J Health Psychol 2009;14:142-51.doi:10.1177/ 1359105308097954

3. Hunt N, McHALE SUE. Reported experiences of persons with Alopecia Areata. Journal of Loss and Trauma 2004;10:33-50.doi:10. 1080/15325020490890633

4. Hunt N, McHale S. The psychological impact of alopecia. BMJ 2005;331:951-3.doi:10.1136/bmj.331.7522.951

5. Rumsey N, Harcourt D. Body image and disfigurement: issues and interventions. Body Image 2004;1:83-97.doi:10.1016/S17401445(03)00005-6

6. Thompson A, Kent G. Adjusting to disfigurement: processes involved in dealing with being visibly different. Clin Psychol Rev 2001;21(5):663-82.doi:10.1016/S0272-7358(00)00056-8

7. Ruiz-Doblado S, Carrizosa A, García-Hernández MJ. Alopecia Areata: psychiatric comorbidity and adjustment to illness. Int J Dermatol 2003;42:434-7.doi:10.1046/j.1365-4362.2003.01340.x

8. Koo JY, Shellow WV, Hallman CP, et al. Alopecia Areata and increased prevalence of psychiatric disorders. Int J Dermatol 1994;33:849-50.doi:10.1111/j.1365-4362.1994.tb01018.x

9. Cash TF. The psychological effects of androgenetic alopecia in men. J Am Acad Dermatol 1992;26:926-31.doi:10.1016/01909622(92)70134-2

10. Cash TF, Price VH, Savin RC. Psychological effects of androgenetic alopecia on women: comparisons with balding men and with female control subjects. J Am Acad Dermatol 1993;29:568-75.doi:10.1016/ 0190-9622(93)70223-G

11. Chiang $Y Z$, Bundy C, Griffiths CE, et al. The role of beliefs: lessons from a pilot study on illness perception, psychological distress and quality of life in patients with primary cicatricial alopecia. $\mathrm{Br} \mathrm{J}$ Dermatol 2015;172:130-7.doi:10.1111/bjd.13259

12. Messenger AG, McKillop J, Farrant $P$, et al. British Association of Dermatologists' guidelines for the management of alopecia areata 2012. Br J Dermatol 2012;166:916-26.doi:10.1111/j.1365-2133. 2012.10955.x

13. Wiggins S, Moore-Millar K, Thomson A. Can you pull it off? appearance modifying behaviours adopted by wig users with Alopecia in social interactions. Body Image 2014;11:156-66.doi:10. 1016/j.bodyim.2014.01.004
14. Welsh N, Guy A. The lived experience of Alopecia Areata: a qualitative study. Body Image 2009;6:194-200.doi:10.1016/j.bodyim. 2009.03.004

15. Cash TF. The psychology of hair loss and its implications for patient care. Clin Dermatol 2001;19:161-6.doi:10.1016/S0738081X(00)00127-9

16. Goffman E. Stigma: notes on the management of spoiled identity. Simon and Schuster 2009.

17. Kacar SD, Soyucok E, Bagcioglu E, et al. The perceived stigma in patients with Alopecia and mental disorder: a comparative study. Int $J$ Trichology 2016;8:135.doi:10.4103/0974-7753.189005

18. Bewley A, Affleck A, Bundy C, et al. Psychodermatology services guidance: the report of the British Association of Dermatologists' Psychodermatology Working Party. Br J Dermatol 2013;168:1149-50 .doi:10.1111/bjd.12330

19. Lavda AC, Webb TL, Thompson AR. A meta-analysis of the effectiveness of psychological interventions for adults with skin conditions. Br J Dermato/ 2012;167:970-9.doi:10.1111/j.13652133.2012.11183.x

20. Macbeth AE, Tomlinson J, Messenger AG, et al. Establishing and prioritising research questions for the treatment of Alopecia Areata: the Alopecia Areata priority setting partnership. Br J Dermatol 2017. doi:10.1111/bjd.15099

21. Connor KM, Davidson JR, Churchill LE, et al. Psychometric properties of the social phobia inventory (SPIN). Brit J Psychiat 2000;176:379-86.

22. Spitzer RL, Kroenke K, Williams JB. Validation and utility of a selfreport version of PRIME-MD: the PHQ primary care study. JAMA 1999;282:1737-44.

23. Kroenke K, Spitzer RL, Williams JB. The phq-9. J Gen Intern Med 2001;16:606-13.

24. Spitzer RL, Kroenke K, Williams JB, et al. A brief measure for assessing generalized anxiety disorder: the GAD-7. Arch Intern Med 2006;166:1092-7.doi:10.1001/archinte.166.10.1092

25. Kroenke K, Spitzer RL, Williams JB, et al. Anxiety disorders in primary care: prevalence, impairment, comorbidity, and detection. Ann Intern Med 2007;146:317-25.doi:10.7326/0003-4819-146-5200703060-00004

26. SPSS: IBM Corp. Released 2013. IBM SPSS Statistics for Windows, Version 22.0. Armonk, NY: IBM Corp.

27. Mayring P. Qualitative content analysis. Qualitative Social Research 2000;1 www.qualitative-research.net/index.php/fqs/article/view/ $1089 / 2385$.

28. Rosman S. Cancer and stigma: experience of patients with chemotherapy-induced alopecia. Patient Educ Couns 2004;52:333-9.doi:10.1016/S0738-3991(03)00040-5

29. Smart Richman L, Leary MR. Reactions to discrimination, stigmatization, ostracism, and other forms of interpersonal rejection: a multimotive model. Psychol Rev 2009;116:365-83.doi:10.1037/ a0015250

30. Clarke A, Thompson AR, Jenkinson E, et al. CBT for appearance anxiety: psychosocial interventions for anxiety due to visible difference. John Wiley \& Sons 2013.Contributors AT and KM developed the protocol for this study. All authors have contributed to the preparation of this manuscript. KM carried out data analysis for quantitative data, and KM and CW analysed qualitative data to ensure reliability of coding. All data were checked by AT.

Funding This work was supported by the Economic and Social Research Council (Grant number ES/J00215/1).

Competing interests None declared.

Patient consent This paper does not contain personally identifiable information regarding any participant.

Ethics approval University of Sheffield Psychology Department Ethics Committee.

Provenance and peer review Not commissioned; externally peer reviewed.

Data sharing statement The protocol and data files are available from the corresponding author on request.

Open Access This is an Open Access article distributed in accordance with the terms of the Creative Commons Attribution (CC BY 4.0) license, which permits others to distribute, remix, adapt and build upon this work, for commercial use, provided the original work is properly cited. See: http://creativecommons.org/ licenses/by/4.0/

(c) Article author(s) (or their employer(s) unless otherwise stated in the text of the article) 2017. All rights reserved. No commercial use is permitted unless otherwise expressly granted. 